LANCASTER-TH 94-14 hep-ph/9408324

\title{
False Vacuum Chaotic Inflation: the New Paradigm?
}

\author{
David H. Lyth ${ }^{\dagger}$ and Ewan D. Stewart* \\ †School of Physics and Materials, \\ University of Lancaster, \\ Lancaster LA1 4YB, U. K. \\ Department of Physics, Kyoto University, Kyoto 606, Japan.
}

\begin{abstract}
Contribution to appear in the proceedings of 'Birth of the Universe and Fundamental Physics' held in Rome, 1994. Presented by D. H. Lyth.
\end{abstract}

Since the observation by COBE of cmb anisotropy at the expected level, the idea that an initial inflationary era sets the conditions for the subsequent hot big bang has enjoyed unprecedented popularity. At the same time, the level of interest in building an actual model of inflation has never been lower. Perhaps the general feeling is that there are already too many theoretically viable models, making it pointless to look for more. Such a perception, if it exists, is far from the truth because in the context of supergravity (now generally accepted within the particle physics community) the possibilities for model building are extremely limited, and largely unexplored. Some recent work is reported here, with particular focus on a remarkable new paradigm for model building [1-10], in which one or more non-inflaton fields are sitting in a false vacuum, whose energy density is supposed to dominate the total.

A model of inflation is defined by giving the Lagrangian of the relevant fields, together with some information about their initial values. Here we focus on Einstein gravity models, noting that modified gravity models can usually be rewritten as Einstein gravity models at the expense of making the matter lagrangian more complicated. During inflation, the energy density is dominated by the scalar field effective potential, and all fields except the inflaton field are constant.

There have been three popular paradigms for Einstein gravity inflation. 'New Inflation' 11] postulates that the inflaton field $\phi$ is in thermal equilibrium before inflation, at a temperature high enough to set $\phi=0$. The minimum of the potential is supposed to correspond to $\phi=\phi_{\min } \neq 0$, and inflation occurs as $\phi$ rolls slowly towards $\phi_{\min }$, ending when $\phi$ starts to oscillate around $\phi_{\min }$. After perhaps a long delay, reheating occurs (ie., $\phi$ decays into other fields which thermalise). The popularity of New Inflation has declined more or less continuously since it was proposed, because model building is difficult, and because this paradigm leaves unresolved the question of what sets the initial conditions for the pre-inflationary hot big bang.

'True Vacuum Chaotic Inflation' [12] postulates that our universe emerges from the Planck scale with its energy density dominated by scalar fields. Their values are large enough that the potential soon dominates spacetime gradient terms. All of them but the inflaton field quickly adjust to their true vacuum values and thereafter the situation is the same as in New Inflation; inflation occurs as the inflaton field $\phi$ rolls slowly towards the vacuum and it ends when $\phi$ oscillates around the vacuum, then after perhaps a long delay 'reheating' occurs . (Of course the term 'reheating' is a misnomer now, because thermal equilibrium is being established for the first time).

'False Vacuum Chaotic Inflation' [1, 3] (also called 'Hybrid Inflation') again postulates initial domination by scalar fields, with all except the inflaton field quickly adjusting to 
minimise the potential. The difference is that one or more of the non-inflaton fields is held in a false vacuum through its coupling to the inflaton field. The false vacuum energy dominates the total, 1 and inflation typically ends only when the false vacuum is destabilized as the inflaton field falls through some critical value $\phi_{c}$. There ensues a phase transition to the true vacuum, which may be of first or second order and which may or may not produce topological defects. (The first order case is discussed by David Wands in these proceedings, and both cases are treated in some detail in $[6]$.)

In global supersymmetry the (F-term) potential is of the form

$$
V=V_{\text {global }} \equiv \sum_{\alpha}\left|\partial W / \partial \phi_{\alpha}\right|^{2}
$$

where the superpotential $W$ is an analytic function of the complex scalar fields $\phi_{\alpha}$. Superpotentials have been constructed in the past which can lead to New or True Vacuum Chaotic inflation [13]. Recently, it was realised [6] that a superpotential already proposed in the literature in the context of particle physics leads naturally to False Vacuum Chaotic Inflation. It is

$$
W=\sigma\left(\Psi_{1} \Psi_{2}+\Lambda^{2} / \sigma\right) \Phi
$$

where $\Phi, \Psi_{1}$ and $\Psi_{2}$ are chiral superfields, $\sigma \lesssim 1$ is a dimensionless coupling and $\Lambda$ sets the energy scale. The corresponding potential is

$$
V=\sigma^{2}\left|\psi_{1} \psi_{2}+\Lambda^{2} / \sigma\right|^{2}+\sigma^{2}\left(\left|\psi_{1}\right|^{2}+\left|\psi_{2}\right|^{2}\right)|\phi|^{2} .
$$

where $\phi, \psi_{1}$ and $\psi_{2}$ are the scalar components of the superfields. For initial values $|\phi|>$ $\left|\psi_{1}\right|,\left|\psi_{2}\right|$ this gives False Vacuum Chaotic Inflation with an absolutely flat potential $V(\phi)=$ $\Lambda^{4}$. A small slope can be provided [6] by giving $\phi$ a soft supersymmetry breaking mass $m \sim 1 \mathrm{TeV}$, and in any case a slope is provided by the one loop correction which is of order [9] $\left(8 \pi^{2}\right)^{-1} \sigma^{2} \Lambda^{4} \ln \left[\left(8 \pi / m_{P l}^{2}\right)^{1 / 2} \phi\right]$.

Globally supersymmetric models of inflation are generally spoiled when supergravity is taken into account [6, 8]. In supergravity the potential Eq. (1) becomes

$$
\begin{aligned}
V & =\exp \left(\frac{8 \pi}{m_{\mathrm{Pl}}^{2}} K\right)\left[\sum_{\alpha, \beta}\left(\frac{\partial^{2} K}{\partial \bar{\phi}_{\alpha} \partial \phi_{\beta}}\right)^{-1} A_{\alpha} \bar{A}_{\beta}-3 \frac{8 \pi}{m_{\mathrm{Pl}}^{2}}|W|^{2}\right] \\
A_{\alpha} & \equiv \frac{\partial W}{\partial \phi_{\alpha}}+\frac{8 \pi}{m_{\mathrm{Pl}}^{2}} W \frac{\partial K}{\partial \phi_{\alpha}} .
\end{aligned}
$$

Here the Kähler potential $K$ is a real function of the scalar fields and their complex conjugates. The canonical form for $\mathrm{K}$ is

$$
K=\sum_{\alpha}\left|\phi_{\alpha}\right|^{2}+\ldots
$$

which gives canonical kinetic terms to lowest order in the expansion about $\phi=0$. The corresponding expansion for the potential is

$$
\begin{aligned}
V & =\left(1+\frac{8 \pi}{m_{\mathrm{Pl}}^{2}} \sum_{\gamma}\left|\phi_{\gamma}\right|^{2}+\ldots\right)\left\{\sum_{\alpha, \beta}\left(\delta_{\alpha \beta}+\ldots\right) B_{\alpha} \bar{B}_{\beta}-3 \frac{8 \pi}{m_{\mathrm{Pl}}^{2}}|W|^{2}\right\} \\
& =V_{\text {global }}\left(1+\frac{8 \pi}{m_{\mathrm{Pl}}^{2}} \sum_{\gamma}\left|\phi_{\gamma}\right|^{2}+\text { other terms }\right)+\text { other terms } \\
B_{\alpha} & \equiv \frac{\partial W}{\partial \phi_{\alpha}}+\frac{8 \pi}{m_{\mathrm{Pl}}^{2}}\left(\bar{\phi}_{\alpha}+\ldots\right) W .
\end{aligned}
$$

This last expression shows that it is difficult to build a supergravity model of inflation,

\footnotetext{
${ }^{1} \mathrm{~A}$ variant is to have the false vacuum energy density negligible compared with the total. As far as inflation is concerned one then has a scenario which is indistinguishable from True Vacuum Chaotic Inflation, but topological defects produced when the false vacuum destabilizes might be cosmologically significant [6].
} 
because it is difficult to satisfy the necessary flatness condition [4] $\left|V^{\prime \prime} / V\right| \ll 8 \pi / m_{P l}^{2}$. If the condition is satisfied in the global supersymmetric limit.2 it will be violated by the supergravity correction that we have exhibited, for a generic inflaton field and generic choices of $W$ and $K$.

How can this problem be avoided? One way is to suppose that the inflaton corresponds to an 'angular' as opposed to a 'radial' degree of freedom, so that $\sum_{\alpha}\left|\phi_{\alpha}\right|^{2}$ is fixed during inflation. The only model so far proposed which achieves this is 'natural' inflation [14, which invokes a sinusoidal inflaton potential generated by instanton effects.

Barring this possibility, inflation can work only if the forms of $W$ and $K$ are such that the contribution to $V^{\prime \prime} / V$ of the exhibited term is cancelled. Suitable forms have been written down for New Inflation [13] and True Vacuum Chaotic Inflation [15, 16], but they generally have no independent motivation, and in particular do not emerge from superstrings. Recently, forms for $W$ have been given that make False Vacuum Chaotic Inflation [6] and New Inflation [17] work with the minimal form for $K$ (no extra terms in Eq. (5)), B but the minimal form also does not emerge from superstrings. However, general conditions on $W$ and forms for $K$ that allow inflation and $d o$ emerge from superstrings have recently been given [6, 8]. The starting point of [8] is the following recipe for ensuring that the potential receives no inflaton-dependent supergravity correction.

Divide the fields $\phi_{\alpha}$ into two sets, $\varphi_{i}$ and $\psi_{n}$, and suppose that there is an $R$ parity ensuring that $W$ is an odd function of the $\psi_{n}$ and $K$ an even function of the $\psi_{n}$. Suppose that during inflation the $\psi_{n}$ are zero (a natural value since the necessary condition $\partial V / \partial \psi_{n}=0$ is then guaranteed by the $R$ symmetry). Then the $R$ parity ensures that during inflation $W=\partial W / \partial \varphi_{i}=0$. Given these conditions, it is easy to show that there are no inflaton dependent corrections to the global supersymmetry potential provided that $K$ is of the form (suppressing subscripts)

$$
K=-\ln [f(\varphi, \bar{\varphi})-\bar{\psi} C(\chi, \bar{\chi}) \psi]+g(\chi, \bar{\chi})+\mathcal{O}\left(\psi^{2}, \bar{\psi}^{2}\right)
$$

where the $\chi_{j}$ are a subset of the $\phi_{i}$ that are constant during inflation (i.e. that do not contain the inflaton), and $C_{m n}$ is a hermitian matrix.

\section{References}

[1] Linde, A., 1990, Phys. Lett. B249, 18.

[2] Adams, F. C. and Freese, K., 1991, Phys. Rev. D 43, 353.

[3] Linde, A. D., 1991, Phys. Lett. B259, 38.

[4] Liddle, A. R. and Lyth, D. H., 1993, Phys. Rep. 231, 1.

[5] Mollerach, S., Matarrese, S. and Lucchin, F., 1993, preprint astro-ph/9309054.

[6] Copeland, E., Liddle, A. R., Lyth, D. H., Stewart, E. D. and Wands, D., 1994, Phys. Rev. D 49, 417.

[7] Linde, A. D., 1994, Phys. Rev. D 49, 748 (1994).

[8] Stewart, E. D., 1994, preprint hep-ph/9405389.

[9] Dvali, G., Shafi, Q. and Schaefer, R., 1994, preprint hep-ph/9406319.

\footnotetext{
${ }^{2}$ The opposite case where inflation does not occur in the global susy limit is considered in [8]

${ }^{3}$ These both use the same cancellation, that occurs if the effective superpotential during inflation is $W=\Lambda^{2} \phi$.
} 
[10] Stewart, E. D., 1994, preprint astro-ph/9407040.

[11] Linde, A. D., 1982, Phys. Lett. 108B. Albrecht, A. and Steinhardt, P. J., 1982, Phys. Rev. Lett. 48, 1220.

[12] Linde, A. D., 1983, Phys. Lett. B129, 177.

[13] Olive, K., 1990, Phys. Rep. 190, 307.

[14] Freese, K., Frieman, J. A. Olinto, A. V., 1990, Phys. Rev. Lett. 65, 3233; Adams, F. C., Bond, J. R. and Freese, K., Frieman, J. A. and Olinto, A. V., 1993, Phys. Rev. D 47,426 .

[15] Goncharov, A. S. and Linde, A. D., 1984, Class. Quant. Grav. 1, L75.

[16] Murayama, H., Suzuki, H., Yanagida, T. and Yokoyama, J., 1993, preprint hepph/9311326 (1993).

[17] Kumekawa, K., Moroi, T. and Yanagida, T., 1994, preprint hep-ph/9405337 (1994). Matsuda, T., 1994, preprint hep-ph/9405432 (1994). 